\title{
Prenatal Administration of Biotin in Biotin Responsive Multiple Carboxylase Deficiency
}

\author{
KARL S. ROTH, ${ }^{(25)}$ WILLIAM YANG, LORRAINE ALLAN, MARY SAUNDERS, \\ ROY A. GRAVEL, AND KRISHNAMURTI DAKSHINAMURTI
}

\begin{abstract}
Division of Biochemical Development and Molecular Diseases, Department of Pediatrics, University of Pennsylvania School of Medicine and Children's Hospital of Philadelphia, Philadelphia, Pennsylvania, USA [K.S.R. and W.Y.]; and Department of Biochemistry, University of Manitoba Faculty of Medicine, Winnipeg, Manitoba, Canada [L.A. and K.D.J; and The Research Institute, Hospital for Sick Children, Toronto, Ontario, Canada [M.S. and R.A.G.]
\end{abstract}

\begin{abstract}
Summary
Biotin responsive multiple carboxylase deficiency was suspected in a third trimester conceptus on the basis of enzymatic confirmation in fibroblasts cultured from an earlier sibling who suffered a demise in the immediate neonatal period. Maternal urinary organic acid profile was normal throughout the final 4 wk of pregnancy. Oral administration of biotin, $10 \mathrm{mg} /$ day to the mother resulted in a 100-fold increase in urinary biotin excretion within 7 days. Urinary biotin excretion over the subsequent 2 wk decreased steadily, suggesting either decreased maternal absorption or increased fetal sequestration.

After the birth of nonidentical twins, cord blood and urinary organic acid profiles of the infants were normal. However, cord blood biotin concentration was 4 to 7 -fold that of normal newborns. Subsequent enzymatic and genetic complementation studies utilizing cultured skin fibroblasts from the infants demonstrated one of them to be affected by the multiple carboxylase defect, although he was clinically and biochemically normal throughout the neonatal period.
\end{abstract}

Thus, prenatal therapy of this inborn enzymatic defect can be safely and effectively accomplished by administration of pharmacologic biotin doses in the last month of pregnancy.

\section{Speculation}

The affected infant's excellent therapeutic response to pharmacologic doses of biotin, administered to the mother, and the absence of adverse effects in the mother and unaffected twin may eliminate the need for prenatal diagnosis in a pregnancy at risk for multiple carboxylase deficiency.

Biotin responsive multiple carboxylase deficiency results from an inborn error of metabolism in which three mitochondrial, biotin dependent enzymes, namely, pyruvate carboxylase, propionyl-CoA carboxylase, and $\beta$-methylcrotonyl-CoA carboxylase, show diminished activity $(3,17,20,21)$. A defect in cytosolic acetyl CoA carboxylase in this disorder has been recently demonstrated by Feldman and Wolf (10). Affected patients present in the newborn period or early infancy with a clinical picture of fulminant metabolic acidosis, an erythematous, exfoliative dermatitis and urinary excretion of a variety of organic acids attributable to the metabolic back-up produced by the carboxylase defects $(5,7,13,16,22)$. Clinical status and enzyme activity in vivo or in cell culture can be restored to normal by biotin supplementation $(16,17,22)$.

Recently, we have reported the posthumous diagnosis of this disorder (17) in a patient initially (15) thought to have $\beta$-methylcrotonyl CoA carboxylase deficiency, a disease first reported by Stokke et al. $(9,19)$. An earlier sibling of this patient had died within 3 days of birth undiagnosed but with a very similar clinical picture. A subsequent pregnancy in this family came to our attention in the 34th gestational wk. Prenatal diagnosis was not attempted because of the very late stage of pregnancy. Nonetheless, because of the previous family history of neonatal onset, we felt it was imperative to attempt prenatal therapy by oral administration of large doses of biotin to the mother. Subsequent delivery of clinically normal twins, one normal and one later shown to be affected, demonstrated the efficacy of prenatal therapy for this disease.

\section{MATERIALS AND METHODS}

Biotin was obtained in pure form from Hoffman-LaRoche Pharmaceuticals (Nutley, N.J.) ${ }^{23}$ and prepared in $10 \mathrm{mg}$ capsules by the hospital pharmacy. Informed consent was obtained from the mother before administration of the vitamin, which was then given as one $10 \mathrm{mg}$ capsule per day from week 35 until delivery.

Blood and urine specimens were collected and stored at $-76^{\circ} \mathrm{C}$. Urinary and plasma organic acids were quantitated by gas-chromatographic technique and identified by mass spectrometry when necessary as previously described $(6,15)$. Biotin levels were determined according to the radioisotopic dilution method of Dakshinamurti and Allan (8). Urinary creatinines were determined colorimetrically (18).

Cultured fibroblasts were grown from the foreskin, which was obtained at circumcision. Assay of the biotin-dependent carboxylases in fibroblasts after growth in biotin containing or biotin deficient culture medium were carried out by the method of Saunders et al. (17). Genetic complementation studies were performed according to the method of Gravel et al. (11) utilizing cell lines from other affected patients. These included an older sibling resulting from an earlier pregnancy in this family (15).

All reagents were purchased from commercial sources and were of the highest purity available.

\section{RESULTS}

Initial consideration was given to the possibility that the stress of pregnancy in an obligate heterozygote might partially unmask the carrier state. However, gas-chromatographic analysis of the mother's urinary organic acid excretion pattern revealed no obvious abnormalities. Because of the large amount of lactate appearing in the initial urine sample, we determined plasma lactate and pyruvate levels, both of which were normal. Subsequent maternal urine samples showed no consistent effect of biotin upon urinary organic acid excretion. The mother reported no untoward effects following initiation of biotin therapy. After an elective operative delivery of normal twin infants, cord bloods and initial urines were examined for abnormalities of organic acid profiles. These profiles were normal, with no elevations of $\beta$-OH-propionate, $\beta$-methylcrotonylglycine or $\beta$-OH-isovalerate (Fig. 1). 
Enzymatic studies were performed using cultured fibroblasts. The specific activities of each of the biotin-dependent, mitochondrial carboxylases were compared under biotin-rich and biotindepleted growth conditions (Table 1). In biotin-depleted medium, the cells of twin B showed virtually complete deficiencies of all three carboxylase activities while the cells of twin A gave values similar to those of the control cells. In biotin-rich medium the carboxylase activities of twin B's cells were restored.

Genetic complementation experiments were done between fibroblasts of the affected patient (twin B) and cells of the affected $\mathrm{sib}$ as well as those from another patient, both of whom had previously been classified as members of the bio complementation group (17). No complementation could be demonstrated between any of these three strains. In contrast, the cells of twin B did complement propionyl-CoA carboxylase deficient mutants of the $p c c A, p c c B$ and $p c c C$ complementation groups. On the basis of these results, we conclude that twin $B$ shows the complete, biochemical manifestations of the biotin responsive multiple carboxylase disorder and can be assigned to bio complementation groups. These results confirm that the patient must be homozygous for the bio mutation despite the normal clinical presentation during the newborn period.
The results of the measurement of biotin in maternal and cord blood sera were compared to normal controls. Unfortunately, the maternal blood at the time of delivery was lost. However, urinary biotin excretion in the mother increased 100-fold after biotin therapy was instituted. These data are shown in Table 2 and indicate that maternal biotin intake led to a 4- to 7-fold increase in cord blood biotin over control levels that were established in samples from five term infants.

\section{DISCUSSION}

Genetic complementation studies and the assay of the biotindependent mitochondrial carboxylases were used to demonstrate a bio mutation in the first affected member of this family $(15,17)$. The fibroblasts of the first patient were found to be biotin responsive and other patients from the same bio complementation group have also been shown to be biotin responsive in vivo. Therefore, we elected to attempt treatment of the present case with biotin in utero since the late gestational age precluded the use of prenatal diagnosis. Justification for this treatment was based upon two primary factors: (1) the urgent need to prevent a reenactment of the immediate neonatal onset of unrelenting and fatal metabolic

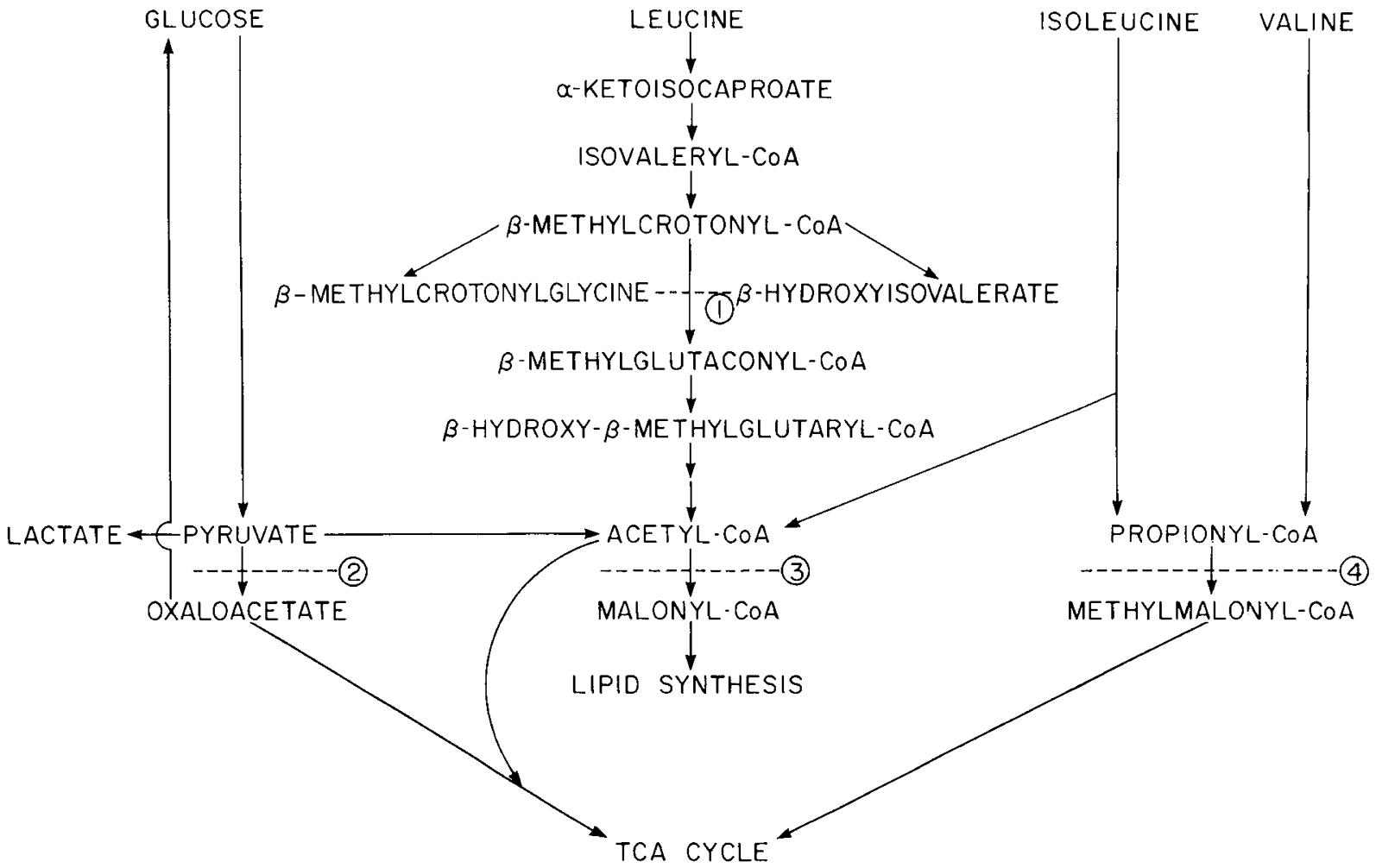

Fig. 1. Metabolic pathways affected in biotin-responsive multiple carboxylase deficiency. The biotin-dependent $\mathrm{CO}_{2}$ fixation reactions are shown numericaliy: (1) $\beta$-methyl-crotonyl CoA carboxylase; (2) pyruvate carboxylase; (3) acetyl CoA carboxylase; and (4) propionyl CoA carboxylase. Enzymes 1,2 and 4 represent the mitochondrial carboxylases known to be deficient in activity in affected individuals. Enzyme 3 (acetyl CoA carboxylase) is a cytosolic enzyme recently shown to have deficient activity in these patients (reference 10).

Table 1. Biotin-dependent carboxylase activity of cultured fibroblasts

\begin{tabular}{|c|c|c|c|c|c|c|}
\hline \multirow{2}{*}{$\begin{array}{l}\text { Cell } \\
\text { line }\end{array}$} & \multicolumn{2}{|c|}{$\begin{array}{l}\text { Propionyl CoA } \\
\text { carboxylase }^{1}\end{array}$} & \multicolumn{2}{|c|}{$\begin{array}{l}\beta \text {-methyl-crotonyl } \mathrm{CoA} \\
\text { carboxylase }^{1}\end{array}$} & \multicolumn{2}{|c|}{ Pyruvate carboxylase ${ }^{1,2}$} \\
\hline & +Biotin & -Biotin & +Biotin & -Biotin & +Biotin & -Biotin \\
\hline Control $^{2}$ & 0.82 & 0.56 & 0.39 & 0.25 & 0.56 & 0.26 \\
\hline Control $^{3}$ & 0.86 & 0.42 & 0.33 & 0.10 & 0.38 & 0.23 \\
\hline Twin A & 1.11 & 0.63 & 0.49 & 0.16 & 2.46 & 1.15 \\
\hline
\end{tabular}

${ }^{1}$ Values represent the means of duplicate determinations; specific activities are expressed as nmoles $/ \mathrm{min} / \mathrm{mg}$ proteins.

${ }^{2}$ Pyruvate carboxylase activities are strain-specific. A broad normal range in fibroblasts has been described (28). 
Table 2. Effect of treatment on blood and urine biotin levels ${ }^{1}$

\begin{tabular}{|c|c|c|}
\hline Serum & $\begin{array}{c}\text { Biotin } \\
(\mathrm{ng} / \mathrm{ml})\end{array}$ & $\begin{array}{c}\text { Biotin } \\
\text { (mg/g creatinine) }\end{array}$ \\
\hline Nongravid females & $\begin{array}{c}10.93 \pm 1.48 \\
(n=5)\end{array}$ & \\
\hline $\begin{array}{l}\text { Normal gravid females } \\
\text { (2nd trimester) }\end{array}$ & $\begin{array}{c}12.53 \pm 0.85^{2} \\
(n=5)\end{array}$ & \\
\hline Mother, no therapy & 10.0 & \\
\hline Cord blood (controls) & $\begin{array}{c}7.34 \pm 0.55^{3} \\
(n=5)\end{array}$ & \\
\hline $\begin{array}{l}\text { Patient, cord blood } \\
\text { Urine }\end{array}$ & 48.75 & \\
\hline $\begin{array}{l}\text { Mother, prebiotin } \\
\quad \text { (gestational wk 34) }\end{array}$ & & 0.0269 \\
\hline $\begin{array}{l}\text { Mother, prebiotin } \\
\quad \text { (gestational wk 35) }\end{array}$ & & 0.0549 \\
\hline $\begin{array}{l}\text { Mother, biotin } \\
\quad \text { (gestational wk 36) }\end{array}$ & & 5.83 \\
\hline $\begin{array}{l}\text { Mother, biotin } \\
\quad \text { (gestational wk 37) }\end{array}$ & & 3.8 \\
\hline $\begin{array}{l}\text { Mother, biotin } \\
\text { (time of delivery) }\end{array}$ & & 2.5 \\
\hline
\end{tabular}

${ }^{1}$ Blood and urine biotin values in mother and patient are given as the means of duplicate determinations. Control values represent the means \pm S.E. of duplicate determinations in five different individuals.

${ }^{2}$ Not significantly different from non-gravid level, $P>0.05$.

${ }^{3}$ Different from normal gravid females, $P<0.001$.

acidosis in two previous successful term pregnancies and, (2) reports in the literature that dosages as high as $40 \mathrm{mg}$ of biotin per day in humans produce no ill effects $(5,7,13,16,22)$.

Maternal blood biotin, in the absence of dietary supplementation, was well within the normal range for pregnant and nonpregnant adult females. These ranges were not significantly different from each other. Our data differ in the latter regard from those of other authors who have reported lower than normal mean biotin levels in pregnant women $(2,4)$ with a gradual drop throughout pregnancy (4). However, these measurements were made using a microbiologic assay system with two different strains of organism and a large $(50-100 \%)$ range of values outside the mean. The radioisotopic dilution assay used for the present determinations provided a rather narrow range of normal values, which maternal urinary biotin levels fell into the normal range reported by Oppel (14) who used a microbiologic assay, which yielded widely variable norms.

Initiation of biotin treatment in the mother resulted in a dramatic increase in urinary biotin excretion. Inasmuch as all maternal urines were randomly collected, urinary biotin levels were calculated on a per gram creatinine basis. Within $1 \mathrm{wk}$, biotin excretion rose by more than two orders of magnitude, into the $\mathrm{mg}$ range, indicating patient compliance. Furthermore, such an increase suggests a significant degree of gastrointestinal absorption of the vitamin, confirming the feasibility of oral administration of pharmacologic doses in prenatal therapy. This is in contrast to the experience of Ampola, et al. (1) during their successful attempt at prenatal therapy of $B_{12}$ responsive methylmalonic aciduria. These authors found it necessary to administer intramuscular $B_{12}$ to achieve serum levels significantly above the control range. Despite the constant biotin dose, maternal urinary biotin excretion fell weekly until delivery. We were unable to explain this, although it could have been due to a number of uncontrolled variables.

Measurement of the cord blood biotin level clearly demonstrated the efficacy of maternal therapy in achieving increased fetal biotin pools. After $3 \mathrm{wk}$ of treatment, the biotin concentration of the affected infant's cord blood had been increased almost 7fold over the mean value determined for five neonates. Inasmuch as the handling of biotin by placental and other biological membranes is poorly understood, it is not possible to infer that larger amounts of the vitamin were present in tissues than in blood. It is possible to state, however, that if simple diffusion alone accounted for entry of biotin into fetal cells, then total body biotin in this infant must have been increased seven times, assuming complete equilibration across all cell membranes. On the other hand, the significantly lower cord blood values as compared with maternal serum levels from normal controls suggests a placental barrier to free diffusion and is consistent with the expected handling of a water soluble substance by a lipid membrane barrier. While this observation is at variance with those of Kaminetzky, et al. (2), who have reported higher circulating biotin levels in fetuses than in gravid females, the microbiologic methodology used by these authors resulted in extremely wide ranges of maternal and cord blood levels which overlap considerably and make interpretation difficult.

Initial examination of the mother's urine did not indicate the presence of any abnormal organic acid. Although the degree of lactate excretion concerned us, a subsequent examination of plasma lactate and pyruvate revealed normal levels of both of these metabolites. Weekly urinary organic acid analyses over the final $5 \mathrm{wk}$ of pregnancy provided further evidence that neither an affected fetus nor the stress of pregnancy in an obligate heterozygote resulted in an abnormal organic acid profile. This is in contrast to the increase in methylmalonic acid excretion by the mother attributed by Ampola, et al. (1) to the affected fetus. Thus, future attempts at prenatal diagnosis of the bio defect must be directed at amniotic fluid cell analyses.

Although enzymatic and genetic complementation studies reported here have confirmed the nature of the genetic defect and shown it to be identical to that of the baby's earlier sibling, this and clinical evidence of the defect were not obtained until 3 months after termination of biotin, at $3 / 12$ months (16). However, it is our view that prenatal biotin therapy probably prevented the immediate perinatal crisis with fatal consequences suffered by our patient's two siblings. Certainly, patients with this inborn error should be treated as early as possible in order to avoid the potential consequences of a later acidotic crisis. The present demonstration of the efficacy and safety of maternal biotin treatment in attaining high fetal levels provides a means for prophylaxis and converts a potentially fatal disease to a minor disorder, as illustrated by this infant's subsequent clinical course.

\section{REFERENCES AND NOTES}

1. Ampola, M. G., Mahoney, M. J., Nakamura, E.. and Tanaka, D.: Prenatal therapy of a patient with vitamin $\mathrm{B}_{12}$-responsive methylmalonic acidemia. New Engl. J. Med., 293: 314 (1975)

2. Baker, H., Frank, O., Thomson, A. D., Langer, A., Munves, E. D., DeAngelis, B., and Kaminetsky, A.: Vitamin profile of 175 mothers and mothers at parturition. Am. J. Clin. Nutr., 28: 56 (1975).

3. Bartlett, K., and Gompertz, D.: Combined carboxylase defect: biotin-responsiveness in cultured fibroblasts. Lancet. 2: 804 (1976).

4. Bhagavan, H. N.: Biotin content of blood during gestation. Inter. J. Vet. Res., 39: 235 (1969).

5. Charles, B. M., Hosking, G., Green, A., Hosking, G., Pollitt, R., Bartlett, K., and Taitz, L. S.: Biotin-responsive alopecia and developmental regression. Lancet, 2: 118 (1979).

6. Cohn, R. M., Updegrove, S., Yandrasitz, J. R., Rothman, R.. and Tomer, K.: Evaluation of continuous solvent extraction of organic acids from biological fluids. Clin. Biochem., 11: 126 (1978).

7. Cowan, M. J., Peckham, S., Wara, D. W., Amman, A. J., Yoshino, M., Sweetman, L., and Nyhan, W.: Multiple biotin-dependent carboxylase deficiencies associated with defects in T-cell and B-cell immunity. Lancet, 2: 115 (1979).

8. Dakshinamurti, K., and Allan, L.: Isotope dilution assay for biotin: use of $\left.{ }^{3} \mathrm{H}\right]-$ biotin. In: Donald B. McCormick, Ed., Lemuel D. Wright, Series Ed.: Methods in Enzymology. pp. 284-287 (Vol. 62) (Academic Press, New York, NY, 1979).

9. Eldjarn, L., Jellum, E., Stokke, O., Pande, H., and Waaler, P. E.: $\beta$-hydroxyisovaleric aciduria and $\beta$-methylcrotonylglycinuria: a new inborn error of metabolism. Lancet. 2: 521 (1970).

10. Feldman, G. L. and Wolf, B.: Deficient acetyl CoA carboxylase activity in multiple carboxylase deficiency. Clin. Chim. Acta, 111: 147 (1981).

11. Gravel, R. A., Lam, K. F., Scully. K. J. and Hsia, Y. E.: Genetic complementation of propionyl-CoA carboxylase deficiency in cultured fibroblasts. Am. J. Hum. Genet., 29: 378 (1977).

12. Kaminetzky, H. A., Baker, H., Frank, O., and Langer, A.: The effects of intravenously administered water-soluble vitamins during labor in normovitaminemic and hypovitaminemic gravidas on maternal and neonatal blood 
vitamin levels at delivery. Amer. J. Obs. Gynecol, 120:697 (1974).

13. Lehnert, W., Niedehoff, H., Junker, A., Saule, H., and Frasch, W.: A case of biotin-responsive 3-methylcrotonylglycine and 3-hydroxyisovaleric aciduria. Eur. J. Pediatr. 132: 107 (1979).

14. Oppel, T. W.: Biotin metabolism in man. Am. J. Med. Sci., 204: 856 (1942).

15. Roth, K. S., Cohn, R., Yandrasitz, J., Preti. G., Dodd, P. and Segal, S.: $\beta$ methylcrotonic aciduria associated with lactic acidosis. J. Pediatr., 88: 229 (1976).

16. Roth. K. S., Yang, W., Foreman, J. W., Rothman, R., and Segal, S.: Holocarboxylase synthetase deficiency: a biotin-responsive organic acidemia. J.Pediatr. 96: 845 (1980).

17. Saunders, M., Sweetman, L., Robinson, B., Roth, K., Cohn, R., and Gravel, R. A.: Biotin-responsive organicaciduria. Multiple carboxylase defects and complementation studies with propionicacidemia in cultured fibroblasts. J. Clin. Invest., 64: 1695 (1979).

18. Thomas, G. H., Howell, R. R.: Hyperuricemia: Uric Acid to Creatinine Ratio. In: George H. Thomas and R. Rodney Howell, Ed.: Selected Screening Tests for Genetic Metabolic Diseases. p. 56 (Year Book Publishers, Chicago, IL, 1973).

19. Stokke, O., Eldjarn, L., Jellum, E., Pande, H., and Waaler, E.: Beta-methylcrotonyl-CoA carboxylase deficiency: a new metabolic error in leucine degrada-

Copyright $\odot 1982$ International Pediatric Research Foundation, Inc. $0031-3998 / 82 / 1602-0126 \$ 02.00 / 0$ tion. Pediatrics, 49: 726 (1972).

20. Sweetman, L., Bates, S. P., Hull, D., and Nyhan, W. L.: Propionyl-CoA carboxylase deficiency in a patient with biotin-responsive 3-methyl-crotonylglycinuria. Pediatr. Res., II: 1144 (1979).

21. Weyler, W., Sweetman, L., Maggio, D. C., and Nyhan, W. L.: Deficiency of propionyl-CoA carboxylase and methylcrotonyl-CoA carboxylase in a patient with methylcrotonylglycinuria. Clin. Chim. Acta, 76: 321 (1977),

22. Wolf, B., Hsia, Y. E., Boychuck, R, Sweetman, L and Nyhan, W. L.: In vivo enzyme activation by biotin of multiple carboxylase deficiency in a neonate (abstract) Pediatr. Res., 14: 529 (1980).

23. Gift of Mr. W. R. Driscoll.

24. Dr. Roch is the recipient of a Research Career Development Award (\#5 K04 HD00257) from the National Institutes of Health.

25. Requests for reprints should be addressed to: Dr. Karl S. Roth, The Children's Hospital of Philadelphia, 34th and Civic Center Blvd., Philadelphia, Pennsylvania 19104.

26. Received for publication February 2, 1981.

27. Accepted for publication August 5, 1981.

28. Raghunathan, R., Russell, J. D., and Arinze, I. J.: Pyruvate carboxylase and phosphoenolpyruvate carboxykinase in cultured human fibroblasts. J. Cell. Physiol., 92: 285 (1977). 